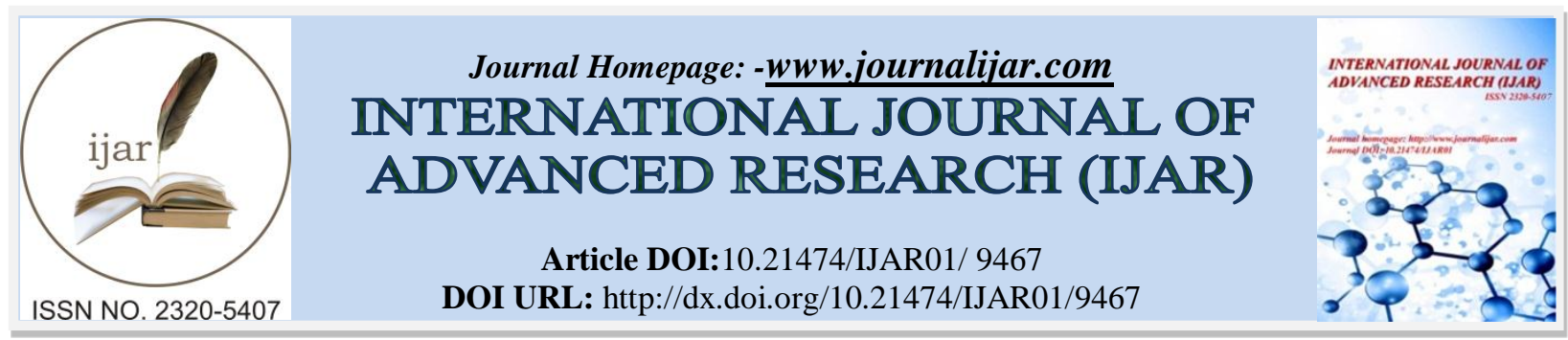

RESEARCH ARTICLE

\title{
WHAT GIVES A SYSTEM APPROACH TO PHYSICS?.
}

Dr. Sc. Techn And prof. V.A. Etkin.

Scientific Research Center of Togliatti State University (Toglyatti, Russian Federation).

\section{Manuscript Info}

\section{Manuscript History}

Received: 02 June 2019

Published: August 2019

\section{Key words:-}

theoretical physics, research methodology, system approach, principle of counterdirectivity, non-
Final Accepted: 04 July 2019 trivial consequences.

\begin{abstract}
It is shown that the emergence of problems in various areas of theoretical physics is systemic in nature and is associated with a violation of the dialectical principle of the opposite orientation of processes in different parts of isolated systems. The fundamental role of this principle is revealed in the system approach adopted as the standard of scientific research. It is shown that the simplification of the study by splitting the object of study into homogeneous parts is inevitably associated with the loss of system-forming bonds and properties that ensure their functioning as a system. Parameters reflecting such properties and characterizing the intensity of the nonequilibrium state are proposed. Using the example of a number of sections of science, it is shown how the consideration of internal relations gives the system under investigation of new properties and radically changes the results of the study.
\end{abstract}

Copy Right, IJAR, 2019,. All rights reserved.

\section{Introduction:-}

The current state of theoretical physics and natural science in general quite clearly demonstrates what can be called a "gnoseological inversion" [1]. It became preferable, in the figurative expression of R. Feynman, "to guess the equations, not paying attention to the physical models or physical explanation" of a particular phenomenon [2]. Speculative constructions and postulates are increasingly replacing experienced facts as a pillar of the modern building of science. Scientists are no longer the fact that their theories do not clarify reality, they no longer set themselves the task of identifying cause-and-effect relationships. The explanation of phenomena ceased to be the main function of science. More and more voices are being heard about the modern crisis of theoretical physics. However, this very rarely offers real ways to overcome this crisis. The purpose of this article is to reveal the role that the principle of antidirection of processes and a systematic approach can play in overcoming it.

The principle of antidirection processes as a methodological basis for a systems approach.

As is known, the basis of the systems approach is the consideration of an object as an integral set of elements with all their inherent connections [1]. Some of these connections are "system-forming", that is, those by which the system as a whole acquires new properties that are absent in any part of it. A particularly vivid example of the emergence of systemic properties is a living organism that loses its ability to function and "self-organize" when it is divided into separate organs. This means that the system-forming properties are not additive. Another example of this nonadditivity can be a heat engine, in the structure of which there is a source, a working body, and a heat receiver. It is enough to deprive the cyclically operating device of any of these elements so that it ceases to function as a thermal or another machine, and becomes a "perpetual motion machine" of the 1 st or 2 nd kind. Thus, it is 
possible to study a heat engine as an energy-transforming system only as a whole as a combination of all three structural elements that are obligatory for it. The same circumstance must be taken into account in the mathematical modeling of a process, for which it is necessary that the system preserves, albeit on a different scale, the same properties. Experienced experimenters know how difficult it is to reproduce on a model the properties of a full-scale object or move from a model to a full-scale object and at the same time preserve all its properties, even if the similarity theory is applied. The fact is that this theory itself is based on differential equations that exclude systemforming properties.

In order to clarify the basic requirements for the object of study in the system approach, we consider an arbitrary set of interacting (mutually moving) material objects that occupy a constant volume $\mathrm{V}$ in space. Any extensive parameter of such a system can be represented as an integral of its local $\rho=d \Theta / d V$ and mean $\bar{\rho}=\Theta / V$ density by the expression $\Theta=\int \rho d V=\int \bar{\rho} d V$. It follows that

$$
\int[d(\rho-\bar{\rho}) / d t] d V=0 .
$$

When $d(\rho-\bar{\rho}) / d t \neq 0$, i.e., if any processes flow in the system, integral (1) vanishes only if the integrand has the opposite sign in different volume elements $\mathrm{dV}$ and is mutually compensated. This position, which we called the "principle of the opposite direction of processes," can be considered as a mathematical expression of the dialectical law of the "unity and struggle of opposites" [3]. The heuristic value of this principle as one of the most general laws of natural science consists in proving that the properties of the system as a whole are not always additive, that is, they are the sum of the properties of its individual parts. These are the "system-forming" properties, which, by definition, are absent in individual parts of the system. In this way, they differ from the extensive properties $\Theta$, which are proportional to the mass $M$, volume $V$, or some other extensiveness factor. Therefore, the terms "extensive" and "additive", often taken for synonyms, are in principle far from equivalent. In particular, in a closed system, the sum of the projections of the internal forces $\mathbf{F}_{i}$ as quantities extensive to any direction is always zero.

Thus, the main feature of the systems approach is the identification and study of "system-based" connections, due to which the object of study acquires new properties that are absent from its parts. In the system analysis of a phenomenon, first of all, it is necessary to single out the parts that undergo opposite changes in the properties of the object of study, the interaction of which, in the final analysis, ensures its functioning as a system. Since any macroprocesses occur only in the absence of equilibrium, in order to preserve the system-forming properties, in no case can one be limited to objects with homogeneous properties in order to avoid the danger of "splashing out the baby with water".

It is hardly necessary to prove how far from such an approach are the fundamental disciplines, which understand as a system just an object of study, isolated from the environment. Particularly indicative in this respect is the Newtonian mechanics, which, as a "first approximation," deliberately excluded from consideration the internal processes in the bodies under study and reduced all changes in their state to movement and acceleration of their mass center. So did quantum mechanics, which considers material points or elementary particles devoid of spatial length and internal structure as an object of study.

It would seem that non-equilibrium thermodynamics, designed to study internal processes, should have avoided this. However, she also resorted to the hypothesis of local equilibrium for this purpose [4]. This hypothesis suggests the presence of equilibrium in the continuum elements (despite the dissipative processes in them), the possibility of describing them with the same set of variables as in the uniform state (despite the appearance of thermodynamic forces), and the validity of on its inevitable transition to inequality). Continuum mechanics uses the same technique, which splits the object of research into an infinite number of "elementary" volumes under the assumption of their homogeneity. Naturally, this way you can dramatically simplify the mathematical model of the phenomenon and describe it by linear differential equations that allow superposition. However, it is no longer possible to restore the "system-forming" properties lost in this way by finding "suitable integrals" due to the non-additivity of such properties [5]. This was, according to A. Poincaré, the cause of "the greatest and deepest shock that physics has experienced since Newton" [6]. 
The foregoing makes it necessary to find a method for studying the properties of non-equilibrium systems without dividing them into conditionally equilibrium parts. This requires non-additive parameters that can describe the nonequilibrium state of the system as a whole. These parameters are suggested by energy dynamics, which is a generalization of the thermodynamics of nonequilibrium processes [7], first to non-static processes of energy transfer and conversion in cyclic and non-cyclic, direct and inverse thermal machines [8], and then to any other forms of energy [9].

As follows from the principle of the opposite direction of processes, any non-equilibrium processes are accompanied by a redistribution of the parameters $\Theta_{i}$ (mass $M$, entropy $S$, the number of moles of the $k$-th substances $N_{k}$, charge 3 , pulse $\boldsymbol{P}$, etc.) over the volume of system $V$. As a result, their density $\rho_{i}=\partial \Theta_{i} / \partial \mathrm{V}$ in different parts of the system varies in the opposite way. In this case, for the thermodynamic description of the state of such systems, it is necessary to introduce additional nonadditive inhomogeneity parameters that characterize the removal of the system from the internal equilibrium (homogeneous) state. Such parameters are the "moments of of the energy carrier" distribution $\mathbf{Z}_{i}=\Theta_{i} \Delta \mathbf{r}_{i}$ with a shoulder $\Delta \mathbf{r}_{i}$, called the "displacement vector" [7]. This non-additive quantity characterizes the shift of the center of the quantity $\Theta_{i}$ from its equilibrium position. Even if one does not resort to decomposition of $\Delta \mathbf{r}_{i}$ into translational, rotational, and vibrational components, the energy of an inhomogeneous system $\mathcal{E}$ becomes a function of twice the number of extensive variables $\mathcal{E}=\mathcal{E}\left(\Theta_{i}, \mathbf{r}_{i}\right)$, the total differential of which can be given the form of identity:

$$
d \mathcal{E} \equiv \Sigma_{i} \Psi_{i} d \Theta_{i}+\Sigma_{i} \mathbf{F}_{i} \cdot d \mathbf{r}_{i}(i=1,2, \ldots, n),
$$

where $\Psi_{i} \equiv\left(\partial \mathcal{E} / \partial \Theta_{i}\right)$ is values of the generalized potentials $\psi_{i}$ (absolute temperature $T$, pressure $p$, the chemical potential of the $k$-th substance $\mu_{k}$, the electric potential of the system $\varphi$, etc.) averaged over the system volume; $\mathbf{F}_{i} \equiv$ $\left(\partial \mathcal{E} \partial \mathbf{r}_{i}\right)$ are forces in their general physical understanding.

Identity (2) is nothing but the result of the joint determination of the basic quantities with which thermokinetic operates. It is valid for any values of the parameters included in it so that their changes do not depend on what caused the change in the state: doing work or relaxation. Due to this, identity (2) is applicable to both reversible and irreversible processes. This makes possible a more general approach to the study of various real processes, without excluding from consideration any (reversible or irreversible) their component. In particular, it allows, without any additional hypotheses or postulates, to introduce the concepts of speed $\mathbf{v}_{i}=d \mathbf{r}_{i} / d t$ and power $d W_{i} / d t$ of any $i$-th process $^{1)}$, as well as fundamental for a number of fundamental disciplines the concept of the flow of $i$-th energy carrier $\mathbf{J}_{i}=d \mathbf{Z}_{i} / d t=\Theta_{i} \mathbf{v}_{i}$ as a generalized speed of the $i$-th vector process (similar to the concept of a mechanical impulse $M \mathbf{v}=d \mathbf{P} / d t)$ and thermodynamic force $\mathbf{X}_{i} \equiv\left(\partial \mathcal{E} / \partial \mathbf{Z}_{i}\right)=\mathbf{F}_{i} / \Theta_{i}$ as a measure of the intensity of the internal state of the system [8]. This gives identity (2) a generality sufficient to not only substantiate all the provisions of the TIP, but also to generalize non-equilibrium thermodynamics to non-thermal processes and machines, and to study its methods of transfer and transformation of any form of energy [9].

This allowed for the synthesis of fundamental disciplines (classical and quantum mechanics, equilibrium and nonequilibrium thermodynamics, hydro and aerodynamics, the theory of heat and mass exchange, electrodynamics and physical kinetics on a single conceptual and methodological basis [10] and taking into account the irreversibility of any real processes. With these are its consequences, which are obtained from identity (2) by using experimentally found conditions of uniqueness, obtained the status of immutable truths.

The objective of this article is a brief overview of the consequences to which the application of the systems approach to the construction of fundamental disciplines based on the principle of opposing processes leads.

\section{Systems approach and classical mechanics.}

The construction of mechanics usually begins with kinematics, which considers the motion of a point in space and time, regardless of the physical causes of this movement. In this case, the concepts of the trajectory of motion, the position on it of a point, its speed and acceleration are introduced purely speculatively. Only then are introduced the concepts of mass and momentum, which are characteristics of a material point, and a transition is made to the study of dynamics, which clarifies the reason why a particular movement occurs in various conditions and what laws it obeys.

\footnotetext{
1) The partial differential symbol đ emphasizes that the elementary work of $₫ W_{i}$ depends on the process path.
} 
At first glance, such a construction of mechanics seems quite natural. However, as L. de Broglie rightly noted [11], this approach is based on the assumption that the results of abstract kinematic consideration can then be extended to the real movement of more complex mechanical objects. Therefore, the "laws" of Newtonian mechanics are nothing more than postulates, not without reason called "definitions" by them. The limitations of these "definitions" are far from always obvious and are often found only when they are considered in a more general range of tasks.

In particular, in the kinematics of a point, the acceleration $\boldsymbol{a} \equiv d \boldsymbol{v} / d t$ is defined as the total time derivative of $\mathrm{t}$ from the velocity vector $v$. Meanwhile, the velocity vector can be changed in two fundamentally different ways: by changing the velocity modulus without changing its direction, and by changing the direction of the velocity vector of the particle without changing its absolute value. These two methods describe clearly distinguishable processes (the first changes the kinetic energy of the particle, the second does not). Therefore, the 2nd Newton's law $F=M a$, formulated before the appearance of vector algebra, extrapolated the concept of acceleration a to a steady rotational motion and led to the concept of centripetal acceleration. This led to the substitution of torque, which accomplishes the work of accelerating the rotational motion, by centripetal, caryolisic, Lorentzian, etc. forces that do not perform work. The negative consequences of such a definition of force are manifested so far and consist, in particular, in the statement about the inevitability of radiation of an electron with its uniform rotation in a circular orbit. A more general definition of force as the energy gradient $\mathbf{F}_{i} \equiv\left(\partial \mathcal{E} / \partial \mathbf{r}_{i}\right)(2)$ excludes the identification of acceleration with reorientation and leads to a completely different interpretation of many phenomena [12].

From the standpoint of a systematic approach, flaws are also found in other initial concepts of mechanics. It is known, for example, that the movement of a separate material point in the absence of any external forces will be rectilinear and uniform (Newton's law of inertia). However, the system of material points can move uniformly not only progressively, but also to rotate "by inertia" with a constant angular velocity. This means that for real bodies the law of inertia should be generalized to rotational motion. Then the possibility of denying the existence of preferential frames of reference, in which the laws of the phenomenon under study look particularly simple and clear, is also excluded.

Generalization requires the 3rd Newton's law (the principle of equality of action and reaction) if it is formulated for the general case of simultaneous action of a set of forces with the resulting $\mathbf{F}_{i}=\Sigma_{j} \mathbf{F}_{j}$. Then immediately it becomes clear the possibility of countering forces of a different nature, the secondary nature of the forces of inertia (reaction) and the redundancy requirements of the directionality of the forces of action and resistance in one straight line, broken, for example, in the lever rule or when the conductors interact with the current. This would avoid a number of contradictions of Newton's 3rd postulate experience.

Thus, with a systematic approach to classical mechanics, the need to correct and generalize all three of Newton's laws is found. This also applies to the law of Newton, which is considered not deductible from any primary principles. If we abandon the concept of "empty" space and proceed from the fact of uneven distribution of mass density in space, it is easy to obtain a more general law of gravity for continuous media, in which not only attraction but also repulsion may occur [13].

The principle of the smallest action, which allows solving many problems from different branches of theoretical physics [14], does not constitute an exception but is still not considered to be deducible from the primary principles. It also turns out to be a consequence of energy dynamics in its application to hydrodynamics and is due to the general orientation of relaxation processes towards equilibrium [15]. Thus, consideration of mechanics as a consequence of the unified theory of the transport and transformation of any form of energy allows us to generalize all its most important propositions.

\section{Systems approach and classical thermodynamics.}

Of all the fundamental disciplines, classical thermodynamics, which adheres to the deductive research method (from the general to the particular) and is based on the general physical principles of the excluded perpetual engine of the 1 st and 2nd kind, most satisfied the requirements of the system approach [16]. Based on these principles, the cycle method made it possible to obtain a huge variety of consequences relating to different areas of knowledge and having, within the limits of applicability, its initial concepts of equilibrium and reversibility, the nature of immutable truths. D. Gibbs's method of characteristic functions (thermodynamic potentials) [17] turned out to be even more general and fruitful. It allowed finding the most important thermodynamic properties of polyvariant systems as derivatives of these functions according to experimentally found independent arguments. 
Difficulties arose only with the release of thermodynamics beyond the fairness of its original concepts of equilibrium and reversibility. For the first time, they manifested themselves in connection with the attempts of thermodynamic analysis of open systems that exchange k-substances with the environment. This process was accompanied by the diffusion of these substances inside the system and a change in its composition, which cannot be reduced either to heat or to work. Some of these difficulties were overcome by Gibbs (1875) by presenting a closed system as a set of open equilibrium subsystems (phases and components), which made it possible to reduce the internal processes of changing the composition of the system to reversible (quasistatic) external energy exchange processes. However, some of these difficulties have been preserved until now and manifested, in particular, in the unsuccessful attempts at thermodynamic resolution of the "Gibbs paradox" - the conclusion that the entropy increases abruptly when non-interacting gases are mixed and that this jump is independent of the nature and degree of difference of these gases [16,18].

Another paradoxical situation arose when thermodynamics was applied to relativistic heat engines (with fast-moving heat sources) and was manifested in the statement that their efficiency is higher than that of a reversible Carnot machine in the same temperature range, as well as recognizing the ambiguity of relativistic transformations of a number of thermodynamic values [16].

A no less paradoxical situation arose when attempts were made to thermodynamically describe spin systems with an inverse population of energy levels, which required the introduction of the notion of negative absolute temperature for such states. This led the researchers to conclude that it is possible to completely transform heat into work in such systems and, on the contrary, to completely transform work into heat, that is, to "inversion" the principle of the excluded perpetual engine of the 2nd kind, which is fundamental to thermodynamics [16,19].

Thermodynamics of irreversible processes (TIP), created by the middle of the 20th century on the basis of L. Onsager's theory by extrapolating classical thermodynamics to nonequilibrium systems with internal irreversible (relaxation) processes [20], did not escape this fate. Difficulties arose primarily in connection with the introduction into the thermodynamics of ideas of transference that were initially alien to her. This required the introduction into thermodynamics of a number of additional hypotheses and postulates. One of such provisions was the already mentioned above hypothesis of local equilibrium I. Prigogine (1960). Another position is connected with the postulation of Onsager's "phenomenological” laws

$$
J_{i}=\Sigma_{j} L_{i j} X_{j}
$$

according to which the generalized speed of some $i$-th process (flow $J_{i}$ ) is determined by all the thermodynamic forces $X_{j}(i, j=1,2, \ldots, n)$, acting in the system, and not their resulting one [19]. In this case, the proof of the symmetry $\left(L_{i j}=L_{j i}\right)$ of the matrix of phenomenological coefficients Lij was carried out not on deterministic bases, but on considerations of a statistical-mechanical nature [20]. All this has deprived the nonequilibrium thermodynamics of completeness and rigor inherent in the classical thermodynamic method. Attempts to overcome these difficulties without any adjustment of the conceptual foundations and the mathematical apparatus of classical thermodynamics were unsuccessful.

The way out was found in the construction of a theory that is in the same relation to classical thermodynamics [16] as the dynamics to static. Such an approach not only confirmed the inviolability of all the laws of classical thermodynamics within the framework of the applicability of its initial concepts of equilibrium and reversibility but also made it possible to correct the basic provisions of the TNP, giving them a strictly phenomenological substantiation. In particular, the Onsager reciprocity relations $L_{i j}=L_{j i}$ turned out to be a consequence of the independence of mixed derivatives on the order of differentiation, and the linear laws (3) assumed the corresponding mechanics form $\mathbf{J}_{i}=L_{i} \mathbf{F}_{i}$, containing the resulting force $\mathbf{F}_{i}=\Sigma_{j} \mathbf{F}_{j}$ [21]. As a result, all the so-called "overlay effects" could be described without recourse to reciprocity relations, expanding the scope of applicability of TIP to nonlinear systems, and further reducing the number of empirical coefficients $L_{i j}$ from $n(n+1) / 2$ to $n$ [22].

\section{System approach and electrodynamics.}

The emergence of electrodynamics is connected, as is well known, with the concept of an electromagnetic field (EMF). At first, Maxwell used it only to designate that part of the space in which magnetic and electric forces were detected. However, later he came to the conclusion that the electromagnetic field (EMF) as a carrier of radiation. In this case, it acquired the properties of a certain medium, which transfers energy "after the radiation left one body and has not yet reached another" [23]. Such a "materialization" of radiation and EMF as its carrier led to its separation from sources and to a conflict with the law of conservation of energy. Indeed, the EMF energy $\mathcal{E}$ is the sum of $\mathcal{E}=$ 
$\varepsilon_{0} \boldsymbol{E}^{2} / 2+\mu_{0} \boldsymbol{H}^{2} / 2$, and with an in-phase change in the strengths of its electric where $\boldsymbol{E}$ and magnetic $\boldsymbol{H}$ components and constancy of the dielectric and magnetic permeabilities $\varepsilon 0$ and $\mu$ o cannot remain constant. This circumstance has not yet received a satisfactory explanation.

In the meantime, the necessity of correcting other Maxwell electrodynamics positions [24], indicating its incompleteness, was revealed. This prompted us to consider electrodynamics as a consequence of energy dynamics in its application to electrical phenomena [9]. With this approach, it turned out that the Coulomb law, like Newton's law, is generated by an uneven distribution in space of electric charges [25]. Moreover, it was also possible to obtain Maxwell's equations as a consequence of energy dynamics under the assumption of the vortex nature of the electric field [26]. He acquired a simple physical meaning and a vector magnetic potential, which turned out to be a function of the angular velocity of rotation of charged particles of matter [27]. This allowed us to supplement identity (2) with terms that take into account the moments $\boldsymbol{M}_{i}$ of the forces $\boldsymbol{F}_{i}$ and establish their connection with the Lorentz forces, thereby bringing electrodynamics closer to electromechanics [28].

However, the most significant changes have undergone views on the nature of light [29]. The existence of radiation, easily penetrating through electromagnetic screens, suggests that electromagnetic radiation is only part of the radiant energy exchange due to oscillations of charged particles. They are not evidence of the electromagnetic nature of light and the experiments of Hertz, since the magnetic component of the flow of radiant energy has not yet been detected, and the radiation is accompanied by the transformation of energy. The opposite direction of the conduction and bias currents, which makes the electric circuit open and the electric field non-vortex, also testifies to the incompleteness of this theory and the electric field [30].

Meanwhile, the approach to the process of radiant energy exchange from the standpoint of identity (2) and the theory of waves convinces that it obeys the same laws as its other types [31], and its non-electromagnetic frequency range, in particular, gravitational recently discovered waves in the cosmic vacuum [32].

\section{The system approach and quantum physics.}

It is even difficult to imagine now that the quantum-relativistic revolution could not have taken place if the theory of irreversible processes and the principle of the opposite direction of processes were known in the early twentieth century when it became necessary to generalize the classical laws of physics to the microworld and megaworld. In particular, the derivation of Planck's radiation law would not require hypotheses contradicting classical physics, if it proceeded not from the concept of equilibrium of a radiator with some materialized "radiation in an absolutely black body cavity", but from non-equilibrium thermodynamics, which considers radiative energy exchange as a combination of non-static wave radiation processes and absorption. Then the radiation quantum would be not a particle, but a single wave, discrete both in space and in time [33].

Otherwise, the situation would have been the situation of the Rutherford-Bohr atom model, if the object of the study was not a single atom, but the entire set of atoms in alternating force fields of any nature. Then it would become obvious that any intra-atomic processes (including the movement of electrons) do not occur without the participation of off-center forces $\mathrm{F}$ emanating from these fields. In this case, Bohr's postulates about the existence of stable (unperturbed) orbits of electrons and about the lack of their duration "jumping" from one stable orbit to another are obviously deprived of their reasons. Then, from the very beginning, it would become obvious that multiphoton emission and "quantum yield" should be taken into account in the photoelectric effect due to the small probability that the number of absorbed photons and emitted electrons is equal. The mysterious quantum numbers would not have been necessary for substantiating the law of the formation of spectral series, as well as the probabilistic interpretation of the Schrödinger wave equation [34]. In this case, the mechanics of the microworld would acquire the character of a consequence of classical physics applied to the wave motion [35].

\section{The system approach and the theory of relativity.}

More than 100 years have passed since the creation of the special and general theory of relativity (STR and GR) [36]. Nevertheless, discussions are still ongoing about the validity of the postulates underlying them and their consequences. One of them was the statement that with an increase in the velocity of the body (system) $\boldsymbol{v}$, parameters such as mass $M$, internal energy $U$, volume $V$, temperature $T$, pressure $p$, entropy $S$, and also the heat $Q$ received by it changes and the work $W$ has done by her. Particularly sharp controversy has been going on, and for more than a dozen years, around the concept of inertial mass. In order to evaluate what is new in the systematic approach to the question, it suffices to consider the whole set of interacting (mutually moving) bodies as an object of 
study. Such a system is by definition closed, and its mass $M$ is unchanged. If the individual bodies of this aggregate changed their mass as their relative motion attenuated or accelerated, the mass of system $M$ would not remain constant in violation of the law of mass conservation. Even more obvious is the question of the invariance of the internal energy of such a system $U$, which by definition does not depend on its movement or position relative to the external environment. As for the temperature $\mathrm{T}$ and pressure $\mathrm{p}$, easily measured by the observer inside the system, their change with the velocity of the center of mass of the system contradicts the Galilean principle of relativity itself, according to which no measurement can distinguish the state of rest and movement [37]. Thus, not only the mass M, determined by I. Newton as a measure of the amount of matter but also any internal parameters of the moving system are a relativistic invariant contrary to the SRT.

Consider now with the same positions GR. It was based on the fact of independence of gravitational and inertial forces from the nature of matter, which A. Einstein formulated in the form of the principle of identity of gravitational and inertial masses. On this basis, he attributed gravity to the properties of space itself and postulated the proportionality of the curvature of space to the mass $M$ of matter contained in it. By virtue of the principle of equivalence of mass and energy, this proportionality also applied to the energy-momentum tensor. Thus, not only geometrical, but also physical properties were attributed to space. In this interpretation, gravity is no longer one of the four types of interaction. This led to the contradiction of GR with quantum physics, which has not yet been overcome.

Meanwhile, the reason for the independence of gravitational and inertial forces from the nature of matter is quite different. This would become obvious if from the very beginning they proceeded from the principle of counterdirectionality of processes and recognize that the carrier of gravitational energy is ether in its Einstein understanding as to the medium filling empty space. As it became known now, this primary (hidden, dark, non-barion) part of the Universe matter is at least $95 \%$ of its mass and participates only in the gravitational interaction. In this case, the gravitational forces $\mathbf{F}_{g} \equiv\left(\partial \mathcal{E} / \partial \mathbf{r}_{g}\right)$, found on the basis of identity (2), depend only on the distribution of the density of primary matter $\rho$, and not on the properties of the substance formed from it.

Finding the gravitational forces of dark matter as a continuum requires a generalization of Newton's law to the case of the absence of "field-forming" and "test" bodies. Such a generalization leads to the law of gravity in the form $\mathbf{X}_{g}$ $=c^{2} \nabla \rho / \rho$, where the intensity of the gravitational field $\mathbf{X}_{g}$ becomes the force of both attraction and repulsion (in accordance with the знаком $\rho$ sign) [38]. Moreover, its magnitude turns out to be many orders of magnitude higher than the specific force of Newton. This circumstance makes condensing primary matter a building material and a source of energy for the synthesis of all chemical elements and all forms of the substance of the Universe. This explains many of the phenomena occurring in it without using A. Einstein's theory of relativity [39].

\section{Systems approach and biophysics.}

To date, enough evidence has accumulated in the natural sciences indicating that not only a destructive but also a creative tendency is inherent in nature. However, classical thermodynamics and thermodynamics of irreversible processes [20] based on the principle of increasing entropy are still attributed to any system, including the Universe as a whole, the desire for "chaos".

The situation changes radically if we take into account the principle of the opposite direction of the processes in biosystems. Then, the inevitability of the occurrence of opposite processes in them along with the vector relaxation processes becomes apparent. These include, in particular, the vector processes of "active transport" of substances (transporting them to an area of increased concentration) and scalar processes, "conjugation" of chemical reactions, when some of them go in the direction of chemical equilibrium, and others in the opposite direction. The latter contributes to the accumulation in the system of "free energy", which determines their "vital activity" [40]. This feature of living organisms is manifested in their ability to perform internal work "against balance", due to which the "self-organization" of biological systems and the ordering of their structure are carried out. However, these processes are anti-dissipative. This gives rise to the "blatant contradiction of thermodynamics with the theory of biological evolution" which compels the explanation of the evolution of biosystems to be the unnatural "origin of order from chaos" [4].

A systematic approach based on the principle of opposing processes eliminates this contradiction. According to him, antidissipative processes are caused by the inner workings performed in the biosystems "against balance" due to the accumulated "free" energy [41]. This energy is replenished by radiation or food consumption so that the distinction 
between objects of animate and inanimate nature is manifested only in the ability of the former to perform along with internal and external work.

The occurrence of antidissipative processes in biosystems slows their approach to equilibrium and extends their productive period [42]. The latter gives an advantage to those of their varieties that have a greater number of degrees of freedom and their corresponding emergent properties. This phenomenon Darwin and interpreted as "the struggle for existence."

\section{System approach and and astrophysics.}

In accordance with the methodology of the systems approach and the principle of the opposite direction of processes, consideration of the Universe as a homogeneous system, the direction of evolution of which in general coincides with the direction of evolution of any part of it, is unacceptable.

This fully applies to cosmology, based on A. Einstein's equations of the general theory of relativity [43]. If the analysis of evolutionary processes given by A. Friedman on the basis of this equation belonged to a homogeneous part of the Universe, the claims of the Big Bang theory to a single scenario of the behavior of the entire infinite Universe would be rejected as groundless from the very beginning [44].

According to the principle of opposing processes, the evolution of some parts and degrees of freedom of the system is carried out at the expense of the involution of others. In the Universe, evolution is manifested in the emergence of baryonic matter and the acquisition of new forms of energy by it and is carried out at the expense of "free" (ordered) energy of primary (non-barion) matter. This process continues there until the pressure of baryon matter in the stars exceeds the compressive forces and causes a "supernova explosion" or even a "big explosion" of the collapsing part of the Universe. In this relatively fast phase, the baryonic substance is dispersed, taking the form of primary matter. This ensures the eternal circulation of the substance of the universe and the balance of baryon and non-barion matter with all their properties.

\section{Conclusion:-}

The undertaken brief overview of the problems in different fields of knowledge shows how useful the construction of various branches of physics on the methodological principles of the systems approach can be, taking into account the counterdirection of non-equilibrium processes. The mere listing of non-trivial consequences of the systems approach [45] convinces us that the causes of the crisis that has arisen in theoretical physics are systemic.

There is no doubt that the development of natural science would have taken a different direction if researchers had the opportunity to slowly understand the causes of the difficulties encountered and take timely measures to correct the paradigm and eliminate them. The above considerations and their detailed elaboration in refereed articles show that this is not so difficult. Then we would not need our belated appeal to return to the classical path of development of physics [46].

The decisive role in the prevention of critical situations in science could be played by international institutions for independent scientific examination of new ideas that are not bound by national, ideological, or corporate interests. These organizations could continuously identify "bottlenecks", formulate problems and contribute to their solution through a system of contests and awards. The spontaneous development of science, which has become a branch of the production of ideas and technologies, leads, as practice shows, to situations similar to world economic crises. 


\section{References:-}

1. Uyomov AI. Systems approach and general theory of systems.- M .: Thought, 1978. (In Russian).

2. Feynman R. Character of Physical Laws.- M.: Physical Encyclopedia, 1984. (In Russian).

3. Etkin V. Principle of non-equilibrium processes counterdirectivity. // Reports by independent authors, 37(2016), 86 - 92). (In Russian).

4. Prigogine I. Time, structure and fluctuations (Nobel lecture in chemistry in 1977) // Uspekhi Fiz. Nauk. 131 (1980). 185-207. (In Russian).

5. Etkin VA. System analysis and modern problems of natural science. // System research and management of open systems. - Haifa, Israel, 3 (2007) .20-26. (In Russian).

6. Poincare A. About science. - M .: "Science", 1983. (In Russian).

7. Etkin VA. Thermodynamics of non-equilibrium processes of energy transfer and transformation. Saratov: SSU Publ. House, 1991. (In Russian).

8. Etkin V. Thermokinetics (Synthesis of Heat Engineering Theoretical Grounds).- Haifa, 2010.

9. Etkin V. Energodynamics (Thermodynamic Fundamentals of Synergetics).- N.Y., 2011.

10. Etkin VA. Synthesis of the fundamentals of engineering disciplines (energodymamic approach to the integration of knowledge).- Saarbrücken: Lamb. Acad. Publ., 2011. (In Russian).

11. De Broglie L. Introduction to Wave Mechanics. //Ann. De Phys, 10(1925).22-24.

12. Etkin VA. Mechanics as a Consequence of Energodynamics. // The Papers of independent Authors.43(2018). 118.

13. Etkin VA. Generalized Law of Gravitation. // World Scientific News, 74 (2017) 272-279).

14. Landau LD, Lifshits EM. Theoretical Physics, V.1-I0. M., Science, 1976. (In Russian).

15. Etkin VA. Energodynamic Substantiation of the Principle Least Action. // World Scientific News, 92(2) (2018) 340-350.

16. Bazarov IP. Thermodynamics. Ed. 4th M., "High School", 1991.

17. Gibbs J. Century Thermodynamic work.(Trans. from English). M.: Gostekhizdat, 1950.

18. Kedrov, BM. The Gibbs Paradox. M .: Science, 1969 (In Russian).

19. Etkin VA. Paralogism of thermodynamics. Saarbrücken: Palm. Ac. Publ., 2015. (In Russian).

20. De Groot SR., Mazur R. Non-Equilibrium thermodynamics. - M.: Mir, 1964. (In Russian).

21. Etkin VA. Uniqueness of driving forces of irreversible processes. //Russian Journal of Physical Chemistry, 63(6).1989. 918-919 (translated from Zhurnal Fizicheskoi Khimii, 63(6).1989. 1660-1662).

22. Etkin VA. Synthesis and new applications of theories of energy transfer and transformation: Diss. ... d-ra tech. sciences. M., 1998. (In Russian).

23. Maxwell JK. Treatise on Electricity and Magnetism. M V.1,2. M.:Science, 1989.

24. Etkin VA. Correction of electrodynamics from the standpoint of energy dynamics. // Reports of independent authors. 34 (2015). 193-208. (In Russian).

25. Etkin VA. Modified Coulomb law. // World Scientific News, 87 (2017) 163-174.

26. Etkin VA. Thermodynamic Derivation of Maxwell's Electrodynamic Equations. //Global Journal of Physics, 3(1).2015.8-13.

27. Etkin VA. On the sense of vector magnetic potential. // Bulletin of the Haifa House of Scientists, 34(2014).7-13. (In Russian).

28. Etkin VA. Correction of Electrodynamics in the Question of the Magnetic Field Work . //Journal of Applied Physics, 9(5), 2017. 71-75. DOI: 10.9790/4861-0905037175.

29. Etkin VA. Paralogism of the electromagnetic theory of light. // Collection of papers. Conf., Israel, Arad, 2015).

30. Etkin VA. To the non-electromagnetic theory of light. // World Scientific News, 80 (2017) 143-157.

31. Etkin VA. On the potential and driving force of radiant heat transfer. // Bulletin of the Haifa House of Scientists, 20 (2010) .2-6. (In Russian).

32. Etkin VA. Basic of the gravitational light theory. // World Scientific News, 81(2) (2017) 184-197 2392-2192

33. Etkin V. Rethinking Plank's radiation law.// Global Journal of Physics, 5(2).2017. 547-553.

34. Etkin VA. Rethinking Quantum Mechanics. //Journal of Applied Physics, 10(2018). 1-8.DOI: 10.9790/4861.

35. Etkin VA. Return physics to the classical path of development. // Problems of Science, 3 (39), 2019. 5-15. (In Russian).

36. Einstein, A. Works on the theory of relativity. M.: Amfora, 2008. ISBN 978-5-367-00842-5.

37. Etkin VA. Independence of mass from speed. //World Scientific News, 72(2017). 150-158.

38. Etkin VA. Energodynamic theory of gravitation. // Aeronautics and Aerospace Open Access Journal, 2019;3(1):40-44. DOI: 10.15406/aaoaj.2019.03.00079 
39. Etkin VA. Alternative of the Theory of Relativity. //Global Journal of Science Frontier Research: A Physics and Space Science, 18(3). 2018. 7-15.

40. Etkin VA. To the non-equilibrium thermodynamics of biological systems. //Biophysics, 40(3)1995. 661-669. (translated from Biofizika, 40(3)1995. 668-676)

41. Etkin VA. Free energy of biological systems. // Biophysics, 48 (4).2003.740-746. (In Russian).

42. Etkin VA. To energodynamic theory of evolution and involution. // Danish Scientific Journal (DSJ), 21(1).2019. 45-50. (In Russian).

43. Etkin VA. System analysis of thermal death and the expanding universe. // Bulletin of the Haifa House of Scientists, 25 (2011) .2-11. (In Russian).

44. Etkin V. Gravitational repulsive forces and evolution of universe. // Journal of Applied Physics, 8(6), 2016. 43-49 (DOI: 10.9790/4861-08040XXXXX).

45. Etkin VA. Return physics to the classical path of development. // Problems of Science, 3 (39), 2019. 5-15. (In Russian).

46. Etkin VA. Nontrivial corollaries of the systems approach in physics. // System research and management of open systems. 2 (2006) .39-44. (In Russian). 